\title{
Significance of estimated salt excretion as a possible predictor of the efficacy of concomitant angiotensin receptor blocker (ARB) and low-dose thiazide in patients with ARB resistance
}

\author{
Hajime Hasegawa ${ }^{1,2}$, Koichi Kanozawa ${ }^{1,2}$, Juko Asakura ${ }^{1,2}$, Kaori Takayanagi ${ }^{1,3}$, Osamu Komuro ${ }^{1}$, \\ Hiroyuki Fukada ${ }^{1}$, Hidetsugu Tokushima ${ }^{1}$, Hisaya Kogure ${ }^{1}$, Masatoshi Matsuzawa ${ }^{1}$ and Tetsuya Mitarai ${ }^{2}$
}

The purpose of this study was to assess the factors affecting the efficacy of combination therapy with losartan and thiazide, with a focus on the significance of salt excretion, via a multicenter observational study. Adult patients with essential hypertension showing therapy resistance to angiotensin receptor blocker (ARB) as a monotherapy or in combination with Ca channel blockers (CCB) were enrolled, and their previously administered ARBs were replaced with the combination tablet containing losartan ( $50 \mathrm{mg}$ per day) and hydrochlorothiazide $(12.5 \mathrm{mg}$ per day). Blood pressure and biochemical parameters were monitored for a year. The baseline blood pressure $(153.4 \pm 14.8 / 86.4 \pm 11.3 \mathrm{~mm} \mathrm{Hg})$ was significantly lowered at the 3rd month $(137.3 \pm 17.4 / 78.2 \pm 11.1 \mathrm{~mm} \mathrm{Hg}, n=93)$ and was maintained at this lower level until the 12th month $(135.3 \pm 14.0 /$ $76.4 \pm 11.1 \mathrm{~mm} \mathrm{Hg}, n=74$ ). The baseline value of estimated salt excretion (eSE), calculated using Tanaka's formula, differed significantly between the high and low treatment response groups, which were defined by the average change in mean blood pressure (MBP-C, $-11.3 \mathrm{~mm} \mathrm{Hg}$; $\mathrm{eSE}=10.8 \pm 2.9 \mathrm{~g}$ per day in high responders vs. $9.2 \pm 2.3 \mathrm{~g}$ per day in low responders, $P=0.004)$. Univariate and multivariate analyses showed a significant correlation between eSE and MBP-C $(R=-0.288$, $P=0.007)$ and indicated the clinical effectiveness of eSE as a possible predictor for MBP-C $(P=0.021)$. In addition, the urine Na-to- $\mathrm{Cr}$ ratio (NCR) demonstrated significant correlations with eSE $(R=0.848, P<0.001)$ and $\mathrm{MBP}-\mathrm{C}(\mathrm{R}=-0.344$, $P<0.001$ ). These results suggest that eSE or NCR could, to a certain extent, predict the efficacy of combination therapy with losartan and low-dose thiazide in patients demonstrating ARB resistance. Combination therapy with losartan and thiazide might thus be suitable for patients with a large amount of salt excretion.

Hypertension Research (2013) 36, 776-782; doi:10.1038/hr.2013.41; published online 25 April 2013

Keywords: angiotensin receptor blocker; salt excretion; salt sensitivity; thiazide

\section{INTRODUCTION}

It has been well established that salt intake is highly related to blood pressure. In general, changes in the body's salt balance should affect the maintenance of blood pressure, ${ }^{1}$ and salt load always induces an increase in blood pressure, even in normal subjects. ${ }^{2}$ The central role of salt balance or intake on the blood pressure is supported by the observation that a majority of the genetic abnormalities that cause hypertension are closely related to the functional abnormalities involved in the excretion of salt from the kidneys. ${ }^{3}$ The role of impaired salt balance in blood pressure elevation is particularly important for Japanese people because the Japanese diet includes large amounts of salt. The recent annual report from the National Institute of Health and Nutrition of Japan showed that the average salt intake in Japanese men and women is 12.0 and $10.3 \mathrm{~g}$ per day, respectively, which are near the highest levels among the developed countries. ${ }^{4}$ Excess salt intake results in salt accumulation in the body, leading to an increase in the extracellular fluid (ECF) volume and resultant intravascular volume overload. ${ }^{5}$ Salt intake or accumulation also generates vascular resistance through cellular atrophy and increased nitric oxide production in the vascular smooth muscle. ${ }^{6,7}$ Furthermore, a high salt intake induces pressure natriuresis to accelerate renal salt excretion. ${ }^{8}$ All of those factors and mechanisms lead to an elevation of blood pressure. Excessive salt intake is also involved in the activation of the renin-angiotensin system in the blood vessels, brain and kidney, ${ }^{9}$ the development of obesity ${ }^{10}$ and insulin resistance, ${ }^{11,12}$ and the activation of TGF- $\beta^{13}$ or NFKB. ${ }^{14,15}$

${ }^{1}$ Saitama Anti-hypertension Losartan-hydrochlorothiazide Trial (SALT) Study Group, Kawagoe, Saitama, Japan; ${ }^{2}$ Department of Nephrology and Hypertension, Saitama Medical Center, Saitama Medical University, Kawagoe, Saitama, Japan and 3Kawagoe Ekimae Clinic, Kawagoe, Saitama, Japan

Correspondence: Dr H Hasegawa, Department of Nephrology and Hypertension, Saitama Medical Center, Saitama Medical University, Kamoda 1981, Kawagoe, Saitama 350-8550, Japan.

E-mail: hase2126@saitama-med.ac.jp

Received 31 October 2012; revised 17 January 2013; accepted 24 January 2013; published online 25 April 2013 
All of those factors also contribute to the elevation of blood pressure. In addition, excess salt intake is known to directly induce blood pressure elevation by increasing the salt concentration in the hypothalamus, which leads to the angiotensin-related activation of the sympathetic nervous system. ${ }^{16,17}$ Thiazide, a diuretic agent that mainly demonstrates salt elimination, could decrease the elevated blood pressure by reducing ECF volume and triggering the additional mechanisms indicated above. Indeed, recent large-scale clinical trials have shown that thiazide is clinically effective for the treatment of hypertension. ${ }^{18}$

Angiotensin receptor blockers (ARBs) are now recommended as first-line antihypertensive agents in a variety of guidelines for hypertension therapy in various countries, including in the latest Japanese guidelines (JSH-2009), because ARBs have superior effects on blood pressure and various organ-protective benefits. ${ }^{19-21}$ However, ARB monotherapy sometimes fails to achieve a sufficient decrease in blood pressure. Some patients who show resistance to ARB monotherapy might exhibit over-consumption or overaccumulation of salt, which would overcome or cancel the effect of ARBs. Such a pathological setting might be particularly prominent among patients with enhanced salt sensitivity. It is known that resistance to $\mathrm{ARB}$ monotherapy is frequently observed in patients with obesity, metabolic syndrome, chronic kidney disease (CKD) or diabetes, and in patients with a high salt intake, all of whom are likely to show enhanced salt sensitivity. ${ }^{19,20}$ Consequently, the coadministration of thiazide to patients who exhibit resistance to ARB monotherapy is a suitable strategy to consider. Indeed, ARB and thiazide combination therapy is recommended in a variety of guidelines for hypertension therapy. ${ }^{19,20}$ The clinical effectiveness of $\mathrm{ARB}$ and thiazide combination therapy would be expected to indicate a correlation between this therapeutic modality and the amount of salt in the body or the amount of salt intake. However, this issue has not been sufficiently analyzed at the clinical level. The present study was designed to study the factors that contribute to decreasing blood pressure by focusing on the correlation between treatment response and salt excretion (or intake) in cases resistant to either ARB monotherapy or combination therapy with an $\mathrm{ARB}$ and a $\mathrm{Ca}$ channel blocker (CCB) in a multicenter cohort study in Saitama Prefecture in Japan (Saitama Anti-hypertension Losartanhydrochlorothiazide Trial: the SALT study group). It is hoped that the results will expand our treatment options for patients with ARB-resistant hypertension.

\section{METHODS}

\section{Study subjects}

This study was conducted at 16 centers participating in the SALT study group (Appendix). The study was performed in accordance with the principles of the Declaration of Helsinki, and the investigational protocol was approved by the Ethics Committee for Human Studies at the Saitama Medical University. Patients who visited one of the participating centers between May 2008 and April 2010, were diagnosed with essential hypertension, and were prescribed an ARB with or without concomitant CCBs for $>1$ month were considered for screening. Among those patients, adult cases who did not achieve the target blood pressure for anti-hypertension therapy described in the 2004 Japanese Society of Hypertension Guidelines for the Management of Hypertension ( $\leqslant 130 / 85 \mathrm{~mm} \mathrm{Hg}$ for young and middle-aged adults and $\leqslant 140 / 90 \mathrm{~mm} \mathrm{Hg}$ for adults older than 75 years $)^{22}$ were considered potential candidates for entry into the trial. All of the enrolled patients provided their informed consent. Patients were excluded from the study if they were taking any type of diuretic or thiazolidinedione and if they exhibited renal insufficiency (serum creatinine $>2.00 \mathrm{mg} \mathrm{dl}^{-1}$ or estimated glomerular filtration rate (eGFR) $<30 \mathrm{ml} \mathrm{min}^{-1}$ ), heart failure (New York Heart Association functional class III or IV for dyspnea at exertion) or severe liver dysfunction.

\section{Study protocol}

After screening, 104 patients were enrolled in the study. Their blood and first morning urine were sampled for baseline biochemical laboratory data, and their ARB regimens were switched to a regimen of concomitant losartan (50 mg per day) and hydrochlorothiazide (HCTZ, $12.5 \mathrm{mg}$ per day) by use of combination tablet. After the medication switch, the enrolled patients were followed up monthly at the individual centers for a 12-month period. The follow-up visits included blood pressure measurement and medical interviews to confirm the absence of adverse effects from the medications. At the 3rd- and 12th-month visits, each patient again provided blood and urine samples for the same measurements that were taken at baseline. During the first 3 months of follow-up, 11 cases were excluded from this study because the patients were not compliant with the medication regimen (4 cases) or because of loss to follow-up due to a move or other causes $(7$ cases). Consequently, 93 participants (38-95 years old) finished the initial 3-month observation. The baseline characteristics of the 93 participants who were eligible for analysis are provided in Table 1, along with the number and average doses of previously prescribed ARBs. During the next 9 months of follow-up, another 19 patients were excluded from the study because they discontinued the medications ( 9 cases), were lost to follow-up ( 9 cases) or withdrew consent ( 1 case); thus the final group consisted of the 74 participants who were successfully followed up for 12 months. Estimated salt excretion (eSE, g per day) was converted from the value of estimated 24-h $\mathrm{Na}$ excretion $(24 \mathrm{HUNaV})$, which was determined using the following equation, proposed by Tanaka et al: ${ }^{23}$

predicted value of 24-h urine $\mathrm{Cr}$ ( $\mathrm{PRCr}$, mg per day)

$=-2.04 \times$ age $+14.89 \times$ body weight $(\mathrm{kg})+16.14 \times$ height $(\mathrm{cm})-2244.45$ $24 \mathrm{HUNaV}($ mEq per day $)=(21.98 \times(\mathrm{uNa} / \mathrm{uCr}) \times \mathrm{PRCr})^{0.392}$

$\mathrm{eSE}(\mathrm{g}$ per day $)=(58.5 \times 24 \mathrm{HUNaV}) / 1000$

\section{Statistical analysis}

All biochemical parameters except brain natriuretic peptide (BNP) and the urine albumin-to-creatinine ratio (ACR) are expressed as the means \pm s.d. As the BNP and ACR values did not show normal (parametric) distribution, they were expressed as median, first and third quartile values. The significance of the difference for continuous variables with parametric distribution was

Table 1 Baseline characteristics and drug usage in patients over a 3-month observation period

\begin{tabular}{lc}
\hline$n$ & 93 \\
Age $($ years $)$ & $67.7 \pm 12.6$ \\
Male $(n)$ & $55(59.1 \%)$ \\
BMI $\left(\mathrm{kg} \mathrm{m}^{-2}\right)$ & $24.6 \pm 3.6$ \\
Obesity $(n(\%))$ & $32(34.4 \%)$ \\
Diabetes $(n(\%))$ & $20(21.5 \%)$ \\
Chronic kidney disease $(n(\%))$ & $21(22.6 \%)$ \\
Dyslipidemia $(n(\%))$ & $41(44.1 \%)$ \\
Cerebrovascular diseases $(n(\%))$ & $15(16.1 \%)$ \\
Acute coronary syndrome $(n(\%))$ & $4(4.3 \%)$
\end{tabular}

Pre-prescribed ARB n (\%)

Average doses (mg per day)

\begin{tabular}{lcc}
\hline Olmesartan & $25(26.9)$ & 20.0 \\
Losartan & $22(23.7)$ & 50.0 \\
Valsartan & $18(19.4)$ & 92.5 \\
Telmisartan & $14(15.1)$ & 38.7 \\
Candesartan & $11(11.8)$ & 7.6 \\
Irbesartan & $3(3.2)$ & 100.0 \\
Total & $93(100)$ & -
\end{tabular}

Abbreviations: ARB, angiotensin receptor blocker; BMI, body mass index. 
determined with a paired $t$-test if the analysis of variance (ANOVA) demonstrated equal distribution, and it was determined with Welch's $t$-test if the ANOVA demonstrated non-equal distribution. Analysis of the mean values of unpaired variables with parametric distribution was made using a $t$-test followed by ANOVA. The significance of paired and unpaired variables with non-parametric distribution was evaluated using Wilcoxon's signed-rank test and Mann-Whitney's $U$-test, respectively. For all of the statistical analyses, we used a microcomputer-assisted program with SPSS (Version 10.0) for Windows Xp (SPSS, Chicago, IL, USA), and $P$-values $<0.05$ were considered significant.

\section{RESULTS}

Figure 1 shows the changes in the average systolic blood pressure (SBP) and diastolic blood pressure (DBP) values in the patients who completed the 12-month observation (Figure 1a), and the average changes in blood pressure from the baseline values are also depicted (Figure 1b). In the 3rd month, both SBP and DBP showed a significant decrease from baseline $(153.4 \pm 14.8 / 86.4 \pm 11.3 \mathrm{~mm} \mathrm{Hg}$ at baseline, $137.3 \pm 17.4 / 78.2 \pm 11.1 \mathrm{mmHg}$ in the 3rd month). However, the blood pressures did not change further over the subsequent 9 months $(135.3 \pm 14.0 / 76.4 \pm 11.1 \mathrm{~mm} \mathrm{Hg}$ in the 12th month), indicating that the significant decrease in blood pressures achieved by the losartan and thiazide combination therapy occurred within the initial 3 months. The blood pressure changes in the 3rd month were $-16.1 \pm 13.6 \mathrm{~mm} \mathrm{Hg}$ for SBP and $7.9 \pm 12.1 \mathrm{~mm} \mathrm{Hg}$ for DBP, as shown in Figure $1 \mathrm{~b}$.

Table 1 shows the baseline characteristics of the enrolled patients who completed the first 3 months of observation (93 cases). The criteria for obesity, diabetes and dyslipidemia were as follows: obesity, body mass index (BMI) $\geqslant 25.0$; diabetes, the use of anti-hyperglycemic medications or fasting blood glucose $>125 \mathrm{mg} \mathrm{dl}^{-1}$, dyslipidemia, the use of lipid-lowering medications or total cholesterol $\geqslant 220 \mathrm{mg} \mathrm{dl}^{-1}$ and/or high-density lipoprotein-cholesterol $\leqslant 40 \mathrm{mg} \mathrm{dl}^{-1}$ and/or triglyceride $\geqslant 150 \mathrm{mg} \mathrm{dl}^{-1}$. The ARBs that the subjects were taking upon enrollment in this study are also listed in Table 1, along with their average doses. Thirty-five patients were concomitantly taking a CCB upon their enrollment in this study; the CCBs used included amlodipine (20 cases, $5.6 \mathrm{mg}$ per day in average), long-acting nifedipine ( 6 cases, $23.3 \mathrm{mg}$ per day), azelnidipine (5 cases, $12.8 \mathrm{mg}$ per day), benidipine (2 cases, $6.0 \mathrm{mg}$ per day), cilnidipine ( 1 case, $10.0 \mathrm{mg}$ per day) and nicardipine ( 1 case, $5.0 \mathrm{mg}$ ).
The time-differential changes in the biochemical parameters of the blood and urine tests are summarized in Table 2. The majority of parameters, including serum $\mathrm{K}$, serum uric acid, blood sugar and hemoglobin $\mathrm{Alc}$, did not show significant differences during the observation period. The eGFR based on a Japanese population ${ }^{24}$ showed a significant decrease at the 3rd month, although there was no significant difference in eGFR between the 3rd and 12th months. The BNP level and ACR also showed significant decreases in the 3rd month compared with their baseline values, and the ACR showed a further significant decrease in the 12th month compared with its value in the $3 \mathrm{rd}$ month.

Mean blood pressure $(\mathrm{MBP}$, shown by $((\mathrm{SBP}+\mathrm{DBP}) \times 2) / 3)$ decreased from $109.6 \pm 10.7 \mathrm{~mm} \mathrm{Hg}$ at baseline to $98.2 \pm 11.1 \mathrm{~mm} \mathrm{Hg}$ in the 3rd month, and the average MBP-change was $-11.3 \pm 11.7 \mathrm{~mm} \mathrm{Hg}$. Based on this value, the enrolled patients were divided into two groups, a high treatment response group (MBPchange $\leqslant-11 \mathrm{~mm} \mathrm{Hg}$ ) and a low treatment response group (MBPchange $>-11 \mathrm{~mm} \mathrm{Hg}$ ), to assess possible contributory factors to the reduction in blood pressure, as shown in Table 3. With the exception of DBP and eSE, none of the parameters were significantly different between the two groups. The eSE value in the low-response group was significantly lower than that in the high-response group, indicating that eSE and baseline DBP might be the only parameters that show a significant difference related to the blood pressure-change induced by combination therapy. Subsequently, the correlation between eSE and the change in MBP was assessed using univariate analysis. The results showed that aside from DBP and MBP, eSE was the only parameter to show a significant correlation with MBP change, as Table 4 shows. The baseline eSE also demonstrated a significant correlation with SBP and DBP change in the 3rd month, as demonstrated in Figures $2 \mathrm{a}$ and $b$. To confirm the significance of eSE as a predictive factor for the efficacy of this combination therapy, multivariate analysis was also applied. Parameters that showed a high probability in the univariate analysis or were presumed to be clinically important were examined for their significance as predictor variables. The analysis showed that eSE was the only factor that significantly predicted a change in MBP, with the exception of baseline DBP (Table 4). Additionally, the difference in parameters between the groups with high and low salt excretion was also studied using the enrolled patients' mean eSE value $(9.95 \pm 2.70 \mathrm{~g}$ per day). In the high eSE group, the MBPchange $(-14.2 \pm 10.6$ vs. $-8.4 \pm 11.3 \mathrm{~mm} \mathrm{Hg}, P=0.013)$ and ACR
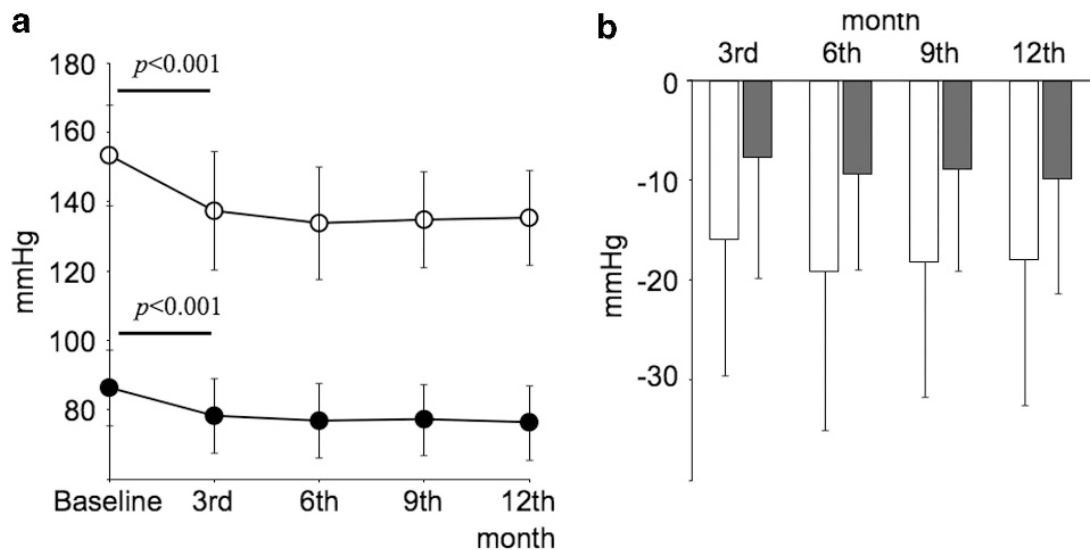

Figure 1 Changes in blood pressure over a 12-month observation period. (a) Changes in the average SBP (open circle) and DBP (closed circle), and (b) changes in SBP (open bar) and DBP (closed bar) from the baseline values in patients who underwent 12 months of observation ( $n=74$ ) are depicted. The results are expressed as the means \pm s.d. DBP, diastolic blood pressure; SBP, systolic blood pressure. 
Table 2 Changes in biochemical parameters

\begin{tabular}{|c|c|c|c|}
\hline & $O M(n=93)$ & $3 M(n=93)$ & $12 M(n=74)$ \\
\hline \multicolumn{4}{|l|}{ Blood test } \\
\hline Albumin $\left(\mathrm{g} \mathrm{dl}^{-1}\right)$ & $4.33 \pm 0.39$ & $4.28 \pm 0.31$ & $4.25 \pm 0.40$ \\
\hline Urea nitrogen $\left(\mathrm{mg} \mathrm{dl}^{-1}\right)$ & $15.5 \pm 4.3$ & $17.1 \pm 5.0^{* *}$ & $16.3 \pm 4.3$ \\
\hline Uric acid $\left(\mathrm{mg} \mathrm{dl}^{-1}\right)$ & $5.73 \pm 1.70$ & $5.63 \pm 1.62$ & $5.89 \pm 1.73$ \\
\hline $\mathrm{Na}\left(\mathrm{mEq} \mathrm{I}^{-1}\right)$ & $141.2 \pm 1.6$ & $140.1 \pm 2.1$ & $140.6 \pm 2.4$ \\
\hline $\mathrm{BNP}\left(\mathrm{pg} \mathrm{ml} \mathrm{l}^{-1}\right)$ & $22.9(10.9,37.7)$ & $16.0(6.7,33.6)^{* *}$ & $14.4(5.4,41.0)^{* *}$ \\
\hline $\mathrm{FBS}\left(\mathrm{mg} \mathrm{dl}^{-1}\right)$ & $119.4 \pm 51.8$ & $110.7 \pm 33.0$ & $117.6 \pm 39.0$ \\
\hline $\mathrm{A} 1 \mathrm{c}(\%)$ & $5.4 \pm 1.1$ & $5.5 \pm 1.0$ & $5.6 \pm 1.1$ \\
\hline \multicolumn{4}{|l|}{ Urine test } \\
\hline Creatinine $\left(\left.g\right|^{-1}\right)$ & $0.84 \pm 0.54$ & $0.80 \pm 0.22$ & $0.91 \pm 0.59$ \\
\hline
\end{tabular}

Abbreviations: ACR, albumin-to-creatinine ratio; BNP, brain natriuretic peptide; eGFR, estimated glomerular filtration rate; FBS, fasting blood sugar; M, months. Note: Values of BNP and ACR are expressed as the median (1st quartile, 3rd quartile).

${ }^{*} P<0.05$ vs. $0 \mathrm{M},{ }^{*} P<0.01$ vs. $0 \mathrm{M}$

$\# P<0.05$ vs. $3 \mathrm{M}, \# \# P<0.01$ vs. $3 \mathrm{M}$

Table 3 Comparative analysis of baseline parameters between high and low responders

\begin{tabular}{|c|c|c|}
\hline & $\begin{array}{l}\text { High responders } \\
\text { (MBP-change } \\
\begin{array}{c}\geqslant-11 \mathrm{~mm} \mathrm{Hg} \\
\mathrm{n}=46)\end{array}\end{array}$ & $\begin{array}{l}\text { Low responders } \\
\text { (MBP-change } \\
\begin{array}{c}<-11 \mathrm{~mm} \mathrm{Hg} \\
\mathrm{n}=47)\end{array}\end{array}$ \\
\hline Age (years) & $66.1 \pm 14.2$ & $69.2 \pm 10.6$ \\
\hline BMI $\left(\mathrm{kg} \mathrm{m}^{-2}\right)$ & $24.6 \pm 3.2$ & $24.5 \pm 4.0$ \\
\hline SBP $(\mathrm{mm} \mathrm{Hg})$ & $157.6 \pm 14.7$ & $152.3 \pm 14.8$ \\
\hline $\mathrm{DBP}(\mathrm{mm} \mathrm{Hg})$ & $93.0 \pm 11.6$ & $80.9 \pm 9.4^{* *}$ \\
\hline Incidence of diabetes $(n)$ & $11(22.0 \%)$ & $9(20.9 \%)$ \\
\hline eGFR $\left(\mathrm{ml} \mathrm{min}^{-1}\right)$ & $85.4 \pm 26.6$ & $77.9 \pm 28.0$ \\
\hline Uric acid $\left(\mathrm{mgdl}^{-1}\right)$ & $5.6 \pm 1.6$ & $5.6 \pm 1.8$ \\
\hline $\mathrm{Na}\left(\mathrm{mEq} \mathrm{I}^{-1}\right)$ & $140.9 \pm 1.8$ & $141.3 \pm 1.6$ \\
\hline $\mathrm{K}\left(\mathrm{mEq} \mathrm{I}^{-1}\right)$ & $4.22 \pm 0.45$ & $4.29 \pm 0.61$ \\
\hline BNP $\left(\mathrm{pg} \mathrm{ml}^{-1}\right)$ & $23.6(10.3,50.3)$ & $22.9(10.9,34.96)$ \\
\hline $\mathrm{A} 1 \mathrm{c}(\%)$ & $5.34 \pm 0.77$ & $5.51 \pm 1.24$ \\
\hline ACR ( $\mu$ g per mg of $\mathrm{Cr}$ ) & $17.1(6.0,53.3)$ & $10.0(5.6,26.8)$ \\
\hline eSE (g per day) & $10.8 \pm 2.9$ & $9.2 \pm 2.3^{*}$ \\
\hline
\end{tabular}

Abbreviations: ACR, albumin-to-creatinine ratio; BMI, body mass index; BNP, brain natriuretic peptide; DBP, diastolic blood pressure; eGFR, estimated glomerular filtration rate; eSE, estimated salt excretion; MBP, mean blood pressure; SBP, systolic blood pressure.

High responders were defined as those with a decline in mean blood pressure of $>11 \mathrm{~mm} \mathrm{Hg}$ over the first 3 months. Values of BNP and ACR indicate median value in addition with 1 st and 3 rd quartile values in the parenthesis as distribution of those values do not show normal distribution.

${ }^{*} P<0.05,{ }^{* *} P<0.01$

(17.7, 7.6, 61.4 vs. 7.5, 4.9, $16.9 \mu \mathrm{g}$ per mgCr, indicating median, $1 \mathrm{st}$ and 3rd quartile values, $P=0.012$ ) were significantly higher than in the low eSE group. The efficacy of the urine Na-to-creatinine ratio (NCR) as a substitutional parameter for eSE was also assessed because eSE calculation using Tanaka's formula is still fairly complex for use in clinical settings. The univariate analysis showed a significantly high
Table 4 Univariate and multivariate analyses the decline of mean blood pressure over a 3-month observation

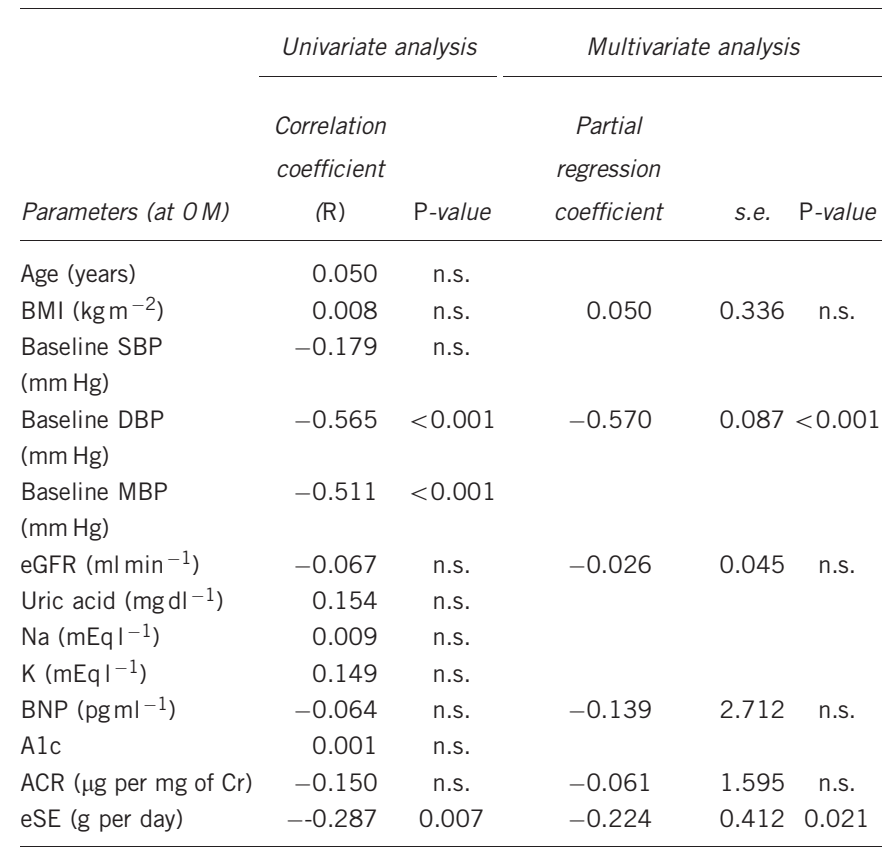

Abbreviations: ACR, albumin-to-creatinine ratio; BMI, body mass index; BNP, brain natriuretic peptide; DBP, diastolic blood pressure; eSE, estimated salt excretion; MBP, mean blood pressure; n.s., not significant; SBP, systolic blood pressure.

Coefficient of determination in this model $=0.42$.

correlation between eSE and NCR, as shown in Figure 2c. As expected from the correlation between NCR and eSE, significant correlation between NCR and MBP change in the 3rd month was also demonstrated by the univariate analysis, suggesting that the NCR was also useful as a predicting parameter of the efficacy of the losartan/thiazide combination therapy. 

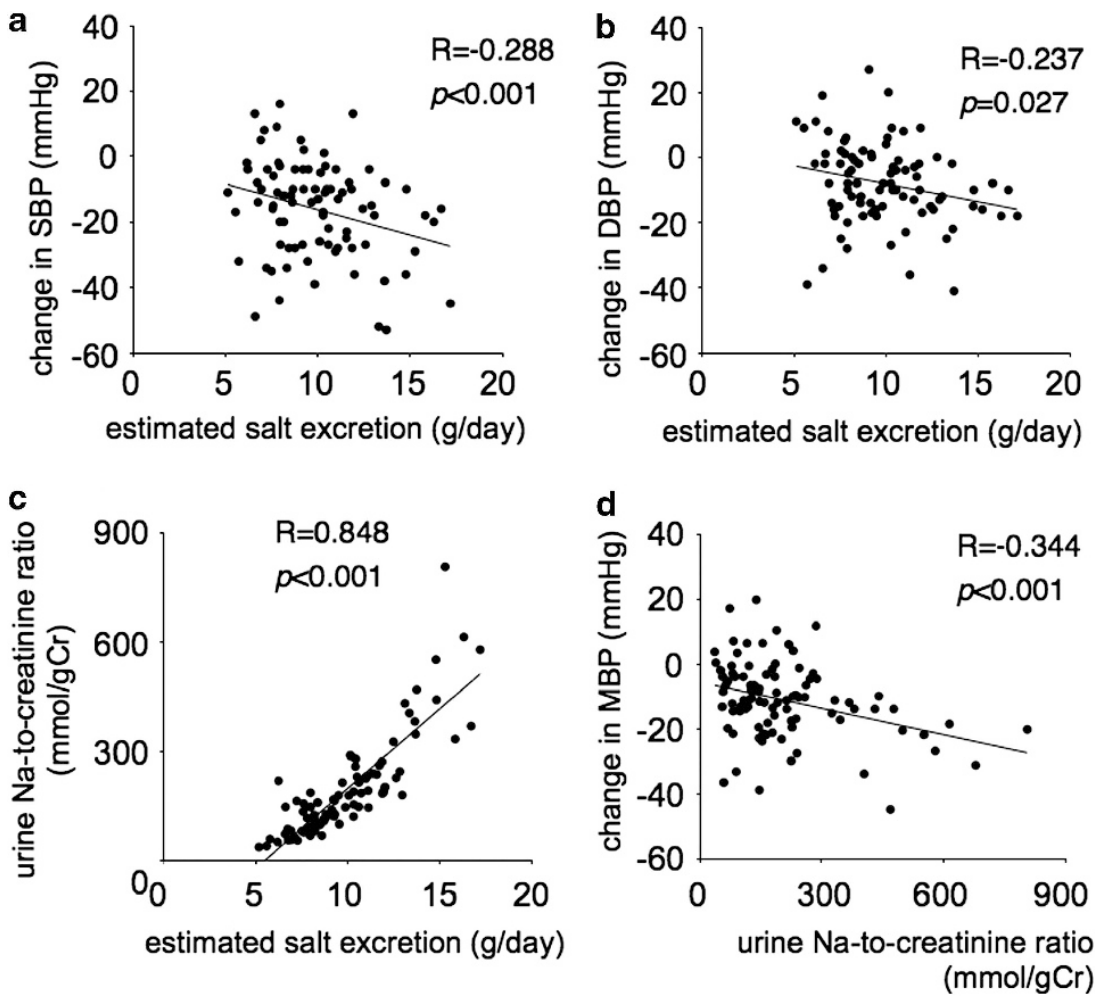

Figure 2 The correlation between estimated salt excretion and changes in systolic or diastolic blood pressure and the correlation between urine Na-to-Cr ratio and estimated salt excretion or changes in mean blood pressure in the 3rd month. The individual correlations between changes in blood pressure during the initial 3 months and baseline eSE were plotted $(n=93)$. The relationships between (a) baseline eSE and SBP changes and (b) baseline eSE and DBP changes were plotted. Correlations between (c) baseline NCR and baseline eSE and (d) baseline NCR and MBP changes were also plotted. $R$ indicates the regression coefficient. Abbreviations: SBP, systolic blood pressure; DBP, diastolic blood pressure; MBP, mean blood pressure; eSE, estimated salt excretion; NCR, urine Na-to-creatinine ratio.

\section{DISCUSSION}

The present study showed that combination therapy with losartan and low-dose hydrochlorothiazide successfully lowered blood pressures in patients whose hypertension was resistant to ARB monotherapy or $\mathrm{ARB}$ and $\mathrm{CCB}$ combination therapy. As the types and doses of preadministered ARBs varied among the individual patients enrolled in this study, it could be considered that the clinical advantages obtained with the combination therapy did not result from the addition of thiazide alone, but from the concomitant use of losartan and thiazide. The present study also revealed that the clinical efficacy of combination therapy with losartan and thiazide was more prominent in patients with high levels of salt excretion, suggesting that the presumed salt intake and the efficacy of the combination therapy are highly correlated. Simultaneously, the correlation between eSE and MBP changes might indicate that more than a few patients with ARB monotherapy- or ARB and CCB combination-resistant hypertension demonstrated thiazide-responsive, salt-sensitive features.

The clinical effectiveness of thiazide has been examined for many years. The Joint National Council (JNC)-7 guideline positions thiazide at the center of antihypertensive therapy. ${ }^{20}$ Similarly, the latest Japanese guideline for hypertension therapy, JSH-2009, recommend that a low dose of thiazide be adopted as a concomitant agent. ${ }^{19}$ Multiple clinical studies have elucidated the potential effects of combination therapy with an ARB and thiazide. ${ }^{25-}$

27 Successful reduction of the proteinuria that remains after ARB monotherapy or ARB-CCB combination therapy has also been reported in a clinical study of patients treated with losartan plus thiazide. ${ }^{28}$ The present study clearly shows that hypertensive patients who showed ARB monotherapy-resistant hypertension demonstrated a significant further decrease in blood pressure and a significant reduction in ACR by switching to losartan and thiazide combination therapy, in agreement with previous studies. The combination therapy is considered to be especially beneficial for preventing thiazide-associated hyperuricemia and ARB-associated hyperkalemia because those adverse effects should be canceled by the losartanassociated acceleration of uric acid excretion and the thiazideassociated acceleration of potassium excretion, respectively. ${ }^{29}$ Indeed, there was no significant change in the serum concentration of potassium and uric acid throughout the entire observation period in this study.

It seems reasonable to consider that the efficacy of thiazide would be at least somewhat correlated with the amount of salt accumulation in the body or the amount of salt intake, although such a correlation has not been directly demonstrated yet. Uzu et al. ${ }^{30}$ demonstrated that the antihypertensive effect of thiazide was more obvious in patients with nocturnal blood pressure elevation who showed a large amount of salt excretion compared with cases without nocturnal blood pressure elevation who showed a smaller amount of salt excretion. Although this study did not show a direct correlation between salt excretion and the effectiveness of thiazide, the relationship was indirectly indicated based on clinical observation. In the present study, the advantage of eSE as a parameter to predict 
the efficacy of losartan and thiazide combination therapy was shown via stratified, univariate and multivariate analyses, although a rough relationship between thiazide effectiveness and salt excretion or intake has been previously discussed only for stratified groups, such as those with high or low salt intake. ${ }^{30,31}$

Multiple formulas have been proposed for estimating salt excretion. However, we considered that it would be difficult to apply these formulas to this study, because some of them require the measurement of lean body mass ${ }^{32}$ or the use of the second urine after awaking as a urine sample. ${ }^{33}$ Tanaka et al. ${ }^{23}$ have reported that sodium excretion for $24 \mathrm{~h}$ could be estimated by the use of urine sodium and $\mathrm{Cr}$ concentration and the estimated $\mathrm{Cr}$ excretion for $24 \mathrm{~h}$, which would be calculated based on the age, body weight and height of individual cases. The Japanese Society of Hypertension recommends using Tanaka's formula. ${ }^{34}$ In the present study, estimated sodium excretion was converted to estimated salt excretion, which was considered a reasonable accurate estimate of the salt intake. Indeed, Tanaka et al. ${ }^{23}$ demonstrated that the estimated salt excretion was highly representative of the salt intake. Therefore, the present study suggests that the effectiveness of combined losartan and thiazide for therapy-resistant hypertension would be significantly affected by salt intake and that the estimation and assessment of salt excretion would be helpful for establishing a strategy for therapy-resistant hypertension.

While salt load causes elevation of blood pressure even in normal subjects, ${ }^{35}$ there are individuals who show an especially pronounced blood pressure elevation in response to salt intake, that is, saltsensitive hypertensives. ${ }^{2}$ It is generally believed that the major clinical features of salt-sensitive hypertension are female sex, obesity, insulin resistance and high incidence of diabetes, renal damage (such as microalbuminuria) and dyslipidemia. ${ }^{2}$ The National Health and Nutrition Survey of Japan reported that the prevalence of obesity and the average BMI in Japan were $30.4 \%$ and 23.1 in males and $20.2 \%$ and 22.3 in females, ${ }^{4}$ whereas those of the patients enrolled in this study were $32.7 \%$ and 24.5 in males and $42.1 \%$ and 24.6 in females, indicating that the study subjects had an obesity prevalence that was higher than that of the Japanese population. Similarly, the enrolled patients also showed a higher prevalence of other parameters, such as dyslipidemia, diabetes and CKD. ${ }^{4}$ These clinical features of the patients in this study match the clinical profile of salt-sensitive hypertension, which might have contributed to the appearance of a correlation between eSE and MBP change in this study. It is presumed that patients with salt-sensitive hypertension basically suffer from an impairment of renal salt excretion, ${ }^{36,37}$ suggesting that their salt intake exceeds their salt excretion. Consequently, a realistic salt intake would be assumed to be more likely than the estimated amount. In any case, patients with salt-sensitive hypertension whose blood pressure is predominantly determined by the salt load or accumulation would be considered resistant to ARB monotherapy, but should respond to the combination of an ARB plus thiazide. Alternately, it might also be suggested that many of the patients with resistance to ARB monotherapy or ARB plus CCB combination therapy might have a higher incidence of salt-sensitive hypertension.

Despite the clinical advantages, daily salt excretion or intake assessments are not realistically straightforward because eSE calculation remains complex in the clinical setting. The present study also showed that NCR might be a more reliable parameter than eSE for estimating daily salt excretion, at least in the patients enrolled in this study. In general, the $95 \%$ reliable range for the average population is determined by the following equation: mean \pm (square root of $D) \times k$, where $D$ indicates the number of samples divided by the variance of population, and $k$ indicates the reliability coefficient (1.96 for $95 \%$ reliability).

Consequently, the $95 \%$ reliable range of eSE in the high-responder group would be from 14.5 to $8.4 \mathrm{~g}$ per day. An analysis of the correlation between eSE and NCR resulted in the following equation:

$\mathrm{NCR}=43.8 \times \mathrm{eSE}-238.9$ ( $\mathrm{mmol}$ per $\mathrm{g}$ of $\mathrm{Cr})$.

Therefore, the $95 \%$ reliable range of eSE in the high-responder group would correspond to 396-134 per g of Cr of NCR. Indeed, one study reported that an NCR of $134 \mathrm{mmol}$ per $\mathrm{g}$ of $\mathrm{Cr}$ might correspond to the salt excretion of Japanese people with average salt intakes, although the study results were based on second urine samples after awaking. ${ }^{34}$ Therefore, the study's clinical analysis of NCR suggests that $\sim 130 \mathrm{mmol}$ per g of Cr or more would be a rough standard for cases that might be expected to show a prominent response to combination therapy with losartan and thiazide.

In conclusion, eSE or NCR could be used to assess the efficacy of losartan and low-dose thiazide combination therapy in patients who demonstrate resistance to ARB monotherapy. Combination therapy with losartan and thiazide might be well suited to patients who show $\mathrm{ARB}$ resistance and high levels of salt excretion.

\section{Limitations}

There were some limitations to this study. First, this study was an observational study in the same population rather than a comparative study. Additionally, the number of enrolled cases was $<100$, and a gender bias existed. These issues raise the possibility that the results obtained in this study are not generally applicable to other populations. However, even in a limited population, the finding of a correlation between estimated salt excretion or intake and efficacy of anti-hypertension therapy using losartan plus thiazide is of clinical importance.

\section{CONFLICT OF INTEREST}

The authors declare no conflict of interest.

\section{ACKNOWLEDGEMENTS}

This work was supported in part by research grants from the Research Foundation for Community Medicine, Utsunomiya, Tochigi, Japan.

1 Guyton AC, Coleman TG, Cowley AV Jr, Scheel KW, Manning RD Jr, Norman RA Jr. Arterial pressure regulation. Overriding dominance of the kidneys in long-term regulation and in hypertension. Am J Med 1972; 52: 584-594.

2 Weinberger MH. Salt sensitivity of blood pressure in humans. Hypertension 1996; 27 (3 Pt 2), 481-490.

3 Juncos R, Hong NJ, Garvin JL. Differential effects of superoxide on luminal and basolateral $\mathrm{Na}+/ \mathrm{H}+$ exchange in the thick ascending limb. Am J Physiol Regul Integr Comp Physiol 2006; 290: R79-R83.

4 National Institute of Health and Nutrition of Japan. Outline for the Results of the National Health and Nutrition Survey Japan, 2007. http:/wwwO.nih.go.jp/eiken/ english/research/project_nhns.html 2007

5 Gavras I, Gavras H. 'Volume-expanded' hypertension: the effect of fluid overload and the role of the sympathetic nervous system in salt-dependent hypertension. J Hypertens 2012; 30: 655-659.

6 Kitiyakara C, Chabrashvili T, Chen Y, Blau J, Karber A, Aslam S, Welch WJ, Wilcox CS. Salt intake, oxidative stress, and renal expression of NADPH oxidase and superoxide dismutase. J Am Soc Nephrol 2003; 14: 2775-2782.

7 Oberleithner H, Riethmuller C, Schillers H, MacGregor GA, de Wardener HE, Hausberg M. Plasma sodium stiffens vascular endothelium and reduces nitric oxide release. Proc Natl Acad Sci USA 2007; 104: 16281-16286.

8 Guyton AC. Blood pressure control-special role of the kidneys and body fluids. Science 1991; 252: 1813-1816.

9 Huang BS, Amin MS, Leenen FH. The central role of the brain in salt-sensitive hypertension. Curr Opin Cardiol 2006; 21: 295-304.

10 He FJ, MacGregor GA. Reducing population salt intake worldwide: from evidence to implementation. Prog Cardiovasc Dis 2010; 52: 363-382. 
11 Ogihara T, Asano T, Ando K, Sakoda H, Anai M, Shojima N, Ono H, Onishi Y, Fujishiro M, Abe M, Fukushima Y, Kikuchi M, Fujita T. High-salt diet enhances insulin signaling and induces insulin resistance in Dahl salt-sensitive rats. Hypertension 2002; 40: 83-89.

12 Kaftan HA, Evrengul H, Tanriverdi H, Kilic M. Effect of insulin resistance on left ventricular structural changes in hypertensive patients. Int Heart J 2006; 47: 391-400.

13 Sanders PW. Salt intake, endothelial cell signaling, and progression of kidney disease. Hypertension 2004; 43: 142-146.

14 Gu JW, Tian N, Shparago M, Tan W, Bailey AP, Manning RD Jr. Renal NF-kappaB activation and TNF-alpha upregulation correlate with salt-sensitive hypertension in Dahl salt-sensitive rats. Am J Physiol Regul Integr Comp Physiol 2006; 291: R1817-R1824.

15 Rodriguez-Iturbe B, Ferrebuz A, Vanegas V, Quiroz Y, Mezzano S, Vaziri ND. Early and sustained inhibition of nuclear factor-kappaB prevents hypertension in spontaneously hypertensive rats. J Pharmacol Exp Ther 2005; 315: 51-57.

16 Mathai ML, Evered MD, McKinley MJ. Central losartan blocks natriuretic, vasopressin, and pressor responses to central hypertonic $\mathrm{NaCl}$ in sheep. Am J Physiol 1998; 275 (2 Pt 2), R548-R554.

17 de Wardener HE, He FJ, MacGregor GA. Plasma sodium and hypertension. Kidney Int 2004; 66: 2454-2466.

18 The ALLHAT Officers and Coordinators for the ALLHAT Collaborative Research Group. Major outcomes in high-risk hypertensive patients randomized to angiotensin-converting enzyme inhibitor or calcium channel blocker vs diuretic: The Antihypertensive and Lipid-Lowering Treatment to Prevent Heart Attack Trial (ALLHAT). JAMA 2002; 288: 2981-2997.

19 Ogihara T, Kikuchi K, Matsuoka H, Fujita T, Higaki J, Horiuchi M, Imai Y, Imaizumi T, Ito S, Iwao H, Kario K, Kawano Y, Kim-Mitsuyama S, Kimura G, Matsubara H, Matsuura H, Naruse M, Saito I, Shimada K, Shimamoto K, Suzuki H, Takishita S, Tanahashi N, Tsuchihashi T, Uchiyama M, Ueda S, Ueshima H, Umemura S, Ishimitsu T, Rakugi H. Japanese Society of Hypertension Committee. The Japanese Society of Hypertension Guidelines for the Management of Hypertension (JSH 2009). Hypertens Res. 2009; 32: 3-107.

20 Chobanian AV, Bakris GL, Black HR, Cushman WC, Green LA, Izzo JL Jr, Jones DW, Materson BJ, Oparil S, Wright JT Jr, Roccella EJ. National Heart, Lung, and Blood Institute Joint National Committee on Prevention, Detection, Evaluation, and Treatment of High Blood PressureNational High Blood Pressure Education Program Coordinating Committee. The Seventh Report of the Joint National Committee on Prevention, Detection, Evaluation, and Treatment of High Blood Pressure: the JNC 7 report. JAMA 2003; 289: 2560-2572.

21 ESH-ESC. 2003. European Society of Hypertension-European Society of Cardiology guidelines for the management of arterial hypertension. J Hypertens 2003; 21: 1011-1053.

22 Japanese Society of Hypertension. Japanese Society of Hypertension guidelines for the management of hypertension (JSH 2004). Hypertens Res 2006; 29 (Suppl), S1-105.

23 Tanaka T, Okamura T, Miura K, Kadowaki T, Ueshima H, Nakagawa H, Hashimoto T. A simple method to estimate populational 24-h urinary sodium and potassium excretion using a casual urine specimen. J Hum Hypertens 2002; 16: 97-103.

24 Irie F, Iso H, Sairenchi T, Fukasawa N, Yamagishi K, Ikehara S, Kanashiki M, Saito $Y$, Ota H, Nose T. The relationships of proteinuria, serum creatinine, glomerula filtration rate with cardiovascular disease mortality in Japanese general population. Kidney Int 2006; 69: 1264-1271.

25 Ito H, Ishii K, Kihara H, Kasayuki N, Nakamura F, Shimada K, Fukuda S, Iwakura K, Yoshikawa J, Effect of ARB/Diuretics on Diastolic Function in Patients with

\section{APPENDIX}

Members of the SALT study group: Masaru Arai (Arai Clinic), Nobuyuki Enomoto (Enomoto Clinic), Hiroyuki Fukada (Fukada Clinic), Hajime Hasegawa (Department of Nephrology and Hypertension, Saitama Medical Center, Saitama Medical University), Shigehisa Inokuma (Inokuma Clinic for Gastro-Intestinal Surgery), Kimio Ito (Ito Clinic), Koichi Kanozawa (Kanozawa Clinic), Kenji Kawashima (Faculty of Internal Medicine, Gyoda General
Hypertension (EDEN) trial investigators. Adding thiazide to a renin-angiotensin blocker improves left ventricular relaxation and improves heart failure in patients with hypertension. Hypertens Res 2012; 35: 93-99.

26 Kita T, Yokota N, Ichiki Y, Ayabe T, Etoh T, Tamaki N, Kato J, Eto T, Kitamura K. One-year effectiveness and safety of open-label losartan/hydrochlorothiazide combination therapy in Japanese patients with hypertension uncontrolled with ARBs or ACE inhibitors. Hypertens Res 2010; 33: 320-325.

27 Palmer BF. Improving BP control with combined renin-angiotensin system blockade and thiazide diuretics in hypertensive patients with diabetes mellitus or kidney disease. Am J Cardiovasc Drugs 2008; 8: 9-14.

28 Vogt L, Waanders F, Boomsma F, de Zeeuw D, Navis G. Effects of dietary sodium and hydrochlorothiazide on the antiproteinuric efficacy of losartan. J Am Soc Nephrol 2008; 19: 999-1007.

29 Hosoya T, Kuriyama S, Ohno I, Kawamura T, Ogura M, Ikeda M, Ishikawa M, Hayashi F, Kanai T, Tomonari H, Soejima M, Akaba K, Tokudome G, Endo S, Fukui A, Gomi H, Hamaguchi A, Hanaoka K, Hara Y, Hara Y, Hasegawa T, Hayakawa H, Hikida M, Hirano K, Horiguchi M, Hosoya M, Ichida K, Imai T, Ishii T, Ishikawa H, Kameda C, Kasai T, Kobayashi A, Kobayashi H, Kurashige M, Kusama $Y$, Maezawa H, Maezawa $Y$, Maruyama Y, Matsuda H, Matsuo N, Matsuo T, Miura Y, Miyajima M, Miyakawa M, Miyazaki Y, Mizuguchi M, Nakao M, Nokano H, Ohkido I, Ohtsuka Y, Okada K, Okamoto $H$, Okonogi $H$, Saikawa $H$, Saito $H$, Sekiguchi $C$, Suetsugu $Y$, Sugano $N$, Suzuki T, Suzuki T, Takahashi H, Takahashi Y, Takamizawa S, Takane K, Morita T, Takazoe K, Tanaka H, Tanaka S, Terawaki H, Toyoshima R, Tsuboi N, Udagawa T, Ueda H, Ueda Y, Uetake M, Unemura S, Utsunomiya M, Utsunomiya Y, Yamada T, Yamada Y, Yamaguchi Y, Yamamoto H, Yokoo T, Yokoyama K, Yonezawa H, Yoshida H, Yoshida M, Yoshizawa T. Antihypertensive effect of a fixed-dose combination of losartan/hydrochlorothiazide in patients with uncontrolled hypertension: a multicenter study. Clin Exp Nephrol 2012 16: 269-278.

30 Uzu T, Sakaguchi M, Yokomaku Y, Kume S, Kanasaki M, Isshiki K, Araki S, Sugiomoto T, Koya D, Haneda M, Kashiwagi A. Effects of high sodium intake and diuretics on the circadian rhythm of blood pressure in type 2 diabetic patients treated with an angiotensin II receptor blocker. Clin Exp Nephrol 2009; 13 300-306.

31 Moser M, Feig PU. Fifty years of thiazide diuretic therapy for hypertension. Arch Intern Med 2009; 169: 1851-1856.

32 Kamata K, Tochikubo O. Estimation of 24-h urinary sodium excretion using lean body mass and overnight urine collected by a pipe-sampling method. J Hypertens 2002; 20 2191-2197.

33 Kawasaki T, Itoh K, Uezono K, Sasaki H. A simple method for estimating 24 h urinary sodium and potassium excretion from second morning voiding urine specimen in adults. Clin Exp Pharmacol Physiol 1993; 20: 7-14.

34 Kawano Y, Tsuchihashi T, Matsuura H, Ando K, Fujita T, Ueshima HWorking Group for Dietary Salt Reduction of the Japanese Society of Hypertension. Report of the Working Group for Dietary Salt Reduction of the Japanese Society of Hypertension (2) Assessment of salt intake in the management of hypertension. Hypertens Res 2007; 30: 887-893.

35 Luft FC, Rankin LI, Bloch R, Weyman AE, Willis LR, Murray RH, Grim CE, Weinberger $\mathrm{MH}$. Cardiovascular and humoral responses to extremes of sodium intake in normal black and white men. Circulation 1979; 60: 697-706.

36 Kimura G, Dohi Y, Fukuda M. Salt sensitivity and circadian rhythm of blood pressure: the keys to connect CKD with cardiovascular events. Hypertens Res 2010, 33: 515-520.

37 Fukuda M, Kimura G. Salt sensitivity and nondippers in chronic kidney disease. Curr Hypertens Rep 2012; 14: 382-387.

Hospital), Hisaya Kogure (Kogure Clinic), Osamu Komuro (Komuro Clinic for Gastro-Intestinal Diseases), Masatoshi Matsuzawa (Matsuzawa Clinic), Toshihiko Nakajima (Nakajima Clinic for Gastro-Intestinal Diseases), Toru Nakamura (Faculty of Internal Medicine, Koseikai Hospital), Tatsuyuki Onodera (Faculty of Cardiovascular Diseases, Nishi-Saitama Chuo National Hospital), Tadaomi Suzuki (Kitamoto Central Clinic), Hidetsugu Tokushima (Tokushima Clinic) 